\title{
EDUCACIÓN FÍSICA CRÍTICA: UN ENFOQUE FUNDAMENTAL PARA LA IGUALDAD DE GÉNERO Y LA INCLUSIÓN EDUCATIVA EN CHILE
}

Critical Physical Education: A fundamental approach to gender equality and educational inclusion in Chile

Educação Física Crítica: Uma abordagem fundamental para a igualdade de gênero e a inclusão educacional no Chile

\author{
Felipe Nicolás Mujica Johnson \\ Universidad Politécnica de Madrid, España \\ fmujica@live.cl
}

\begin{abstract}
Resumen
En la actualidad se están desarrollando diferentes debates y discusiones en torno a la justicia social y la pertinencia de una perspectiva crítica de la educación, que contribuya a la emancipación del alumnado. O en otras palabras, a sus posibilidades de comprender las ideologías que hay detrás de las diferentes marcas o sentidos hegemónicos en la sociedad, como por ejemplo, capitalismo y patriarcado en la Educación Física. En este sentido, el objetivo de este artículo es analizar el fenómeno de la igualdad de género y la inclusión educativa en la asignatura, con énfasis en la situación de Chile. Así, destaca el enfoque mecanicista del currículo como una barrera para la transformación social, la pasividad del profesorado frente a la problemática y a los hombres como los principales reproductores de aquella cultura patriarcal. Finalmente, se considera que el profesorado debe ser formado rigurosamente en la materia y que debe diferenciar los contenidos de la asignatura en función de los estereotipos sociales que se encuentran asociados.

Palabras clave: Educación Física; neoliberalismo; pedagogía crítica; currículo escolar; justicia social.
\end{abstract}

\begin{abstract}
Currently, different debates and discussions are taking place around social justice and the relevance of a critical perspective on education that contributes to the emancipation of
\end{abstract}


students. Or in other words, to its possibilities to understand the ideologies that are behind the different hegemonic brands or senses in society, such as, for example, capitalism and patriarchy in Physical Education. In this sense, the objective of this article is to analyze the phenomenon of gender equality and educational inclusion in the subject, with emphasis on the situation in Chile. Thus, the mechanistic approach of the curriculum stands out as a barrier to social transformation, the passivity of teachers facing the problem and men as the main reproducers of that patriarchal culture. Finally, it is considered that the teaching staff must be rigorously trained in the subject and that they must differentiate the contents of the subject according to the social stereotypes that are associated.

Key words: Physical Education; neoliberalism; critical pedagogy; school curriculum; social justice.

\section{Resumo}

Atualmente, diferentes debates e discussões estão ocorrendo em torno da justiça social e da relevância de uma perspectiva crítica sobre a educação que contribui para a emancipação dos estudantes. Ou, em outras palavras, às suas possibilidades de compreender as ideologias que estão por trás das diferentes marcas ou sentidos hegemônicos na sociedade, como, por exemplo, capitalismo e patriarcado na Educação Física. Nesse sentido, o objetivo deste artigo é analisar o fenômeno da igualdade de gênero e inclusão educacional no tema, com ênfase na situação no Chile. Assim, a abordagem mecanicista do currículo se destaca como uma barreira para a transformação social, a passividade dos professores frente ao problema e os homens como os principais reprodutores dessa cultura patriarcal. Por fim, considera-se que o corpo docente deve ser rigorosamente treinado no assunto e que deve diferenciar o conteúdo do assunto de acordo com os estereótipos sociais que estão associados.

Palavras-chave: Educação Física; neoliberalismo; pedagogia crítica; currículo escolar; justiça social. 


\section{Introducción}

El enfoque de la pedagogía crítica se caracteriza por un carácter moral que rechaza las injusticias sociales, las cuales se encuentra legitimadas en el diseño político de la sociedad. Así, este enfoque exige al profesorado "pensar en una forma de vida académica en la que el punto central del proceso de formación considera esencialmente para quién, por qué, cómo, cuándo y dónde se desarrollan determinadas actividades y ejercicios académicos" (Ramírez, 2008, p. 9). Por ello, se le conoce por ser una mirada educativa que se sustenta en principios éticos y políticos. Por lo mismo, para mejorar los aspectos críticos que aquejan al alumnado en los contextos pedagógicos formales, como la desigualdad de género o la discriminación en torno a la sexualidad, el nivel socioeconómico o las necesidades educativas especiales, es fundamental que la ciudadanía se informe, reflexione y se vincule a estas temáticas educativas. Así, para las transformaciones socioculturales en el ámbito pedagógico, es necesario que las personas "participen constantemente en el dominio político, rehaciendo siempre las estructuras sociales, económicas, en que se dan las relaciones de poder y se generan las ideologías" (Freire, 2001, p. 13). No obstante, la realidad es que una gran parte de la ciudadanía se ha quedado al margen de los procesos políticos, como es el caso de Chile que, tras una larga dictadura cívico-militar, el tejido social quedó bastante dañado y se suma al deterioro de la educación pública, como consecuencia de las políticas neoliberales que se instalaron en aquel trágico periodo.

Este escenario ha generado que en las últimas décadas en Chile, se hayan desarrollado diversas movilizaciones estudiantiles y docentes, para defender el derecho a una educación pública gratuita y de calidad, por lo que se ha exigido modificar la legislación educativa y las condiciones laborales del profesorado. Este escenario coincide con lo que describió en su tiempo Giroux (1999), señalando que el profesorado en las últimas décadas ha tenido que enfrentar una encrucijada ideológica, debido a que el desarrollo profesional docente, ha adoptado un lenguaje tecnocrático y corporativo, el cual extrae el componente político de la educación, así como de la cultura, con la finalidad de tener un mayor control de la población y así mismo, menor resistencia para desarrollar políticas neoliberales. En este sentido, la educación en el siglo XXI ha sido un campo de batalla ideológica, pero como ha 
sido costumbre, con desigualdades de poder entre quienes defienden y atacan la educación pública, motivo por el cual se aprecia a nivel global en la sociedad, una notable expansión de las políticas pedagógicas neoliberales, lo cual afecta las remuneraciones, la temporalidad de horas lectivas y no lectivas, las vacaciones, la infraestructura, los materiales educativos, las relaciones laborales, las relaciones pedagógicas y por lo tanto, el desarrollo de todas las didácticas específicas (Chomsky, 2003; Oliva, 2008; Díez, 2010; Puiggrós, 2010; Apple, 2011; Vicente, 2013; Moreno y Gamboa, 2014; Oliva y Gascón, 2016; Moreno, Toro y Gómez-Gonzalvo, 2018).

Ante este contexto, cobra sentido la lucha pedagógica para transformar el sistema educativo y reivindicar la enseñanza como un espacio social de emancipación o liberación de la ciudadanía (Freire, 1975, 2001). Consecuente con estas ideas, existen educadores y educadoras que asumen un rol crítico de su labor, por lo que demuestran su disconformidad con el currículo establecido, que suele caracterizarse por su desvinculación con las principales problemáticas de la sociedad. Por consiguiente, este profesorado siente la esperanza de transformar la realidad, ya que "la esperanza representa una práctica de demostración, un acto de imaginación moral que permite al profesorado y a otros trabajadores y trabajadoras culturales pensar y actuar de la misma manera” (Giroux, 1999, p. 55).

Con respecto a la función que cumplen los organismos nacionales e internacionales en torno al currículo educativo de las naciones, Cavieres y Apple (2016), señalan que realizan una labor de legitimación del conocimiento y de una significación ideológica que reproduce el poder existente en la sociedad, destacando a la OCDE, como uno de los organismo con alto impacto, por su injerencia en las políticas públicas de educación. Asimismo, es posible señalar que esta legitimación del conocimiento a través de las escuelas, tienen un carácter complejo, ya que no es una legitimación explicita, donde se le prohíba al profesorado asumir un carácter crítico, sino que se le ocupa gran parte de su tiempo pedagógico en asuntos tecnocráticos. Así, el profesorado destinaría forzadamente muy poco tiempo a la formación integral del alumnado y a su propia formación permanente. En otras palabras, se satura al profesorado para disminuir sus posibilidades de contribuir a la emancipación 
social del alumnado. Por esa razón, Vicente (2013), plantea que una EF crítica, requiere ser más que una alternativa metodológica, lo cual a su expresa la dificultad que eso requiere, porque sería incoherente pensar una EF crítica desde un currículo tradicional.

En cuanto a la asignatura de Educación Física (EF), se reconoce que en diferentes países ha sido abordada con una perspectiva acrítica, basada en una aparente neutralidad, ignorando que sus contenidos se encuentran fuertemente significados por las ideologías presentes en la sociedad, como el capitalismo (Devís, 2008; Carter-Thuillier y Moreno, 2017). En este sentido, esta asignatura ha sido víctima de las políticas educativas de carácter global, las cuales se orientan al desarrollo económico de cada país y definen las habilidades que debe desarrollar el alumnado para el ámbito laboral. Esta situación encuentra su cumbre en la globalización del test estandarizado Programme for International Student Assesment (PISA), el cual ha tenido algunas consecuencias lamentables, como el desarrollo de una educación descontextualizada, la hegemonía curricular de algunas disciplinas, la simplificación del proceso pedagógico en los medios de comunicación y por lo tanto, la difusión de una verdad superficial sobre lo que sucede en los centros educativos (Rivas, 2015).

Otra ideología que tiene un carácter transversal en la sociedad y afecta al estudiantado en los entornos pedagógicos, como el de EF, es la del patriarcado. Esta poderosa interpretación de la realidad, ha configurado por largo tiempo una cultura androcéntrica (Carrillo, 2017), utilizando diferentes estructuras sociales (Academia, Deporte, Religión, Política, Arte, etc.). Así, el desarrollo de aquella cultura ha otorgado diferentes privilegios al hombre por sobre la mujer, reproduciendo estereotipos de género (masculinidad y feminidad hegemónica) (Curieses, 2017) y discriminando la diversidad sexual, lo que a su vez propicia las conductas homofóbicas o transfóbicas (Piedra, Rodríguez, Ries y Ramírez, 2013). En el caso de América Latina, este fenómeno no puede ser interpretado sin destacar el proceso de colonización, que negó la cosmovisión de las culturas indígenas e instaló por medio de la violencia una cosmovisión euro centrista (Moreno et al., 2018), la cual se basaba en una estructura patriarcal, pero con un matiz particular, que era el de la iglesia católica romana. En Chile, todavía están muy presentes los rastros de aquella forzosa evangelización, que 
entre muchas formas, se reproduce por medio del control de la corporalidad en los centros educativos o mejor dicho, de las normativas sexistas que restringen o permiten, la construcción corporal del alumnado. Entre aquellas normativas, se encuentra la prohibición a los hombres de tener su pelo largo o de utilizar pendientes, adornos o maquillaje a diferencia de las mujeres. Por otro lado, en diferentes instituciones escolares a las mujeres se les obliga a usar faldas o una vestimenta institucional de un color especial (delantal rosado). De esta forma, los estereotipos corporales se encontrarían aún institucionalizados en forma de habitus que reflejarían la dominación masculina en la sociedad (Bourdieu, 2000). Cabe destacar, que cada centro educativo en Chile determina su reglamento interno de presentación personal, por lo que se aprecia una mirada tradicional del profesorado que decide aquellas normativas, lo cual se podría explicar en la complejidad que implica trascender aquellos sentidos comunes, ya que "conlleva la reproducción de todo un imaginario social que implica una educación diferencial en base al género, es decir, en base a las inscripciones mujer o varón” (Curieses, 2017, p. 66).

Por consiguiente, para que la escuela se configure como un espacio de emancipación ante las ideologías que se encuentran implícitas en la sociedad y se exprese en contra de los poderes que pretenden alienar a la población, es necesario que se realicen "cambios profundos en el campo educativo, así como en las representaciones existentes respecto a “educación”, “escuela”, “estudiantes" y las relaciones que se configuran dentro del aula, para así avanzar hacia una pedagogía transformadora, basada en el dialogo y la cooperación" (Carther-Thuillier y Moreno, 2017, p. 107). No obstante, para que aquellos cambios involucren activamente a las bases educativas (alumnado, profesorado, familias), es importante acompañarlos de una nutrida reflexión y discusión académica, que propicie la comprensión de aquellos fenómenos.

En base a lo mencionado, se ha decidido elaborar un ensayo que aborde algunos aspectos del fenómeno del patriarcado en la EF escolar, con énfasis en el contexto chileno. Entonces, en una primera instancia se abordará el contexto chileno de EF, para reflexionar sobre sus límites epistemológicos. Mientras que en una segunda instancia, se analizará el fenómeno 
de la igualdad de género y la inclusión educativa en la asignatura, con la finalidad de identificar algunas propuestas pedagógicas que contribuyan a la transformación social.

\section{El caso de la EF en Chile}

El currículo de la EF chilena (EFCh) ha sido ampliamente analizado desde una perspectiva crítica y postmoderna (Moreno, Marlen, Almonacid, y Vargas, 2013; Moreno, Rivera y Trigueros, 2014; Moreno, Gamboa y Poblete, 2014; Mujica y Concha, 2015; CartherThuillier y Moreno, 2017; Moreno, 2018), lo que ha desvelado su fuerte carácter positivista, acrítico, tecnocrático y biomédico. Lo anterior queda muy bien reflejado en el nuevo nombre que ha asumido la asignatura, denominada Educación Física y Salud (EFyS) y en el sistema que se ha establecido para medir su calidad, lo cual reduce la asignatura al progreso de la condición física. En este sentido, una EF de calidad no tendría mayores diferencias que un entrenamiento físico realizado por un instructor o preparador físico, anulando la justificación filosófica de la asignatura, que la orienta hacia el desarrollo integral del alumnado, de modo que debería atender la corporeidad y la motricidad de los aprendices en toda su complejidad (Sergio, 1999; Toro, 2006; Toro, 2007; Toro y Valenzuela, 2012; Moreno et al., 2018; Mujica, 2018).

Estos antecedentes, permiten comprender que esta asignatura tiene un rol pasivo ante los sentidos patriarcales que integran sus contenidos, como los estereotipos de género y la heterenormatividad. Precisamente el bloque de contenidos que puede resultar más conflictivo para el desarrollo de una educación no sexista es el del deporte, que desde los antiguos juegos olímpicos, viene propiciando un dominio de los hombres en las competencias atléticas. No obstante, es preciso mencionar que en la antigüedad han existido algunas excepciones culturales en torno al patriarcado en el deporte, debido a que en la antigua civilización cretense pre-helénica (3000-200 a.C), como en la polis de Esparta, el atletismo femenino era un contenido común en la formación de las mujeres (Andrónicos et al., 2003). Por lo tanto, es un error no situar adecuadamente el deporte en la EF, el cual tiene una fuerte carga de sentidos socioculturales (Fernández-Balboa, 1993). Una vez que se ha tomado conciencia de lo presente que se encuentra el sexismo en las actividades físico-deportivas, podría resultar incomprensible que un aspecto tan evidente como el de los 
estereotipos de género discurra en forma tan desapercibida. Pero eso es consecuencia del habitus que se genera, derivando en una normalización de aquellas desigualdades. No obstante, quienes sufren aquella violencia simbólica, quedan con una huella afectiva, marcada por el miedo, como se describe en el estudio de Vidiella, Herraiz, Hernández y Sancho (2010), donde los jóvenes que experimentan aquella masculinidad hegemónica, marcada por la rudeza, temen dañar al género femenino por su supuesta débil naturaleza o quienes no se identifican con aquella masculinidad, temen que los acusen de ser un hombre gay, por todo el acoso y la humillación que ello significa, de modo que intentan parecerse lo más que puedan a la fortaleza masculina.

La despreocupación por esta temática también queda reflejada en el ámbito científico, ya que en Chile, como en Sudamérica, son muy escasos los estudios que aborden las emociones del alumnado en la asignatura de EF (Mujica, Orellana y Concha, 2017), lo cual además, debería abordarse desde una perspectiva sociocultural y cualitativa de la emoción, para de esta forma identificar los diversos significados que se encuentran implícitos, entre los que se podría descubrir la influencia del patriarcado. Del mismo modo, son muy escasos los estudiados que aborden la temática del género, la sexualidad y la discriminación en la EFCh. Ante este panorama, queda en evidencia la necesidad de continuar la reflexión epistemológica sobre la formación del profesorado de EF en Chile y más importante aún, que aquella reflexión cuente con la participación del profesorado que se encuentra en ejercicio a nivel pre-escolar, escolar y universitario. Cabe mencionar, que también es importante visibilizar los aspectos que resultan limitantes para la discusión académica en el país. Uno de ellos, es el precario desarrollo de las revistas científicas chilenas en torno a la EFCh o a las Ciencias de la Actividad Física y del Deporte, lo cual de acuerdo con Moreno y Poblete (2015), representa el poco "desarrollo científico de la EFCh, pero también es motivo de preocupación al no tener una serie de evidencias científicas y pedagógicas contextualizadas al ámbito nacional que den sustento al desarrollo profesional de todos aquellos que se dedican a la asignatura" (p. 291). Esta realidad es muy distinta a la que existe en otras naciones Iberoamericanas, como por ejemplo, Brasil o España, donde el área mencionada ha tenido un mayor desarrollo académico-científico. No obstante, a su vez es 
similar a la de otras naciones de la región, como Argentina, México, Perú o Bolivia, que presentan un escazo desarrollo en el área.

Sin duda que para trascender las limitaciones epistemológicas de la asignatura, que hunden el área de la EFCh en perspectivas poco actualizadas y la dotan de un currículo de corte epistemológico acrítico y mecanicista, es que se requiere continuar con el activismo político-pedagógico, científico-pedagógico y filosófico-pedagógico. En el mismo sentido, Toro y Valenzuela (2012), plantean que para avanzar hacia una EF que vaya más allá de la dimensión física, es fundamental conseguir "una reformulación conceptual, programática y epistémica, proceso, por cierto, que requiere de mucho rigor y generosidad" (p. 229). Asimismo, es preciso que aquella reformulación curricular se realice en todos los niveles de la educación formal y le otorgue mayor importancia a las evaluaciones no tradicionales (evaluación formativa, autoevaluación, evaluación compartida, evaluación cualitativa). Y justamente, que aquellas evaluaciones sirvan para incluir sistemáticamente el desarrollo de la dimensión afectiva-social, que por cierto, por consecuencia de la concepción dualista, es una de las más ignoradas y necesarias para humanizar la sociedad (Moreno-Doña, Trigueros-Cervantes y Rivera-García, 2013; Mujica y Orellana, 2018). Por esa razón, para realizar una reformulación rigurosa, es fundamental que tanto los departamentos de EF encargados de la formación del profesorado, como los responsables políticos de la EF en el país, incorporen en su innovación curricular, las aportaciones científicas de la última década en torno a la construcción epistemológica e histórica-cultural de la EFCh (Toro y Valenzuela, 2012; Moreno y Poblete, 2015).

\section{Posibilidades para transformar los sentidos patriarcales en EF}

A pesar de que la producción científica en torno a la temática en la EFCh sea reducida, será posible en este apartado complementarla con estudios de otros países. Es sabido que el último filtro que tiene el diseño del acto pedagógico en las escuelas es el del profesorado, que por medio de su autonomía puede intencionar diferentes metas de aprendizaje que se encuentren asociadas a las establecidas en el currículo oficial. $\mathrm{O}$ en otras palabras, puede hacerse cargo del currículum oculto que se encuentra presente en la asignatura, de modo que puede propiciar la igualdad de género y la inclusión educativa, aunque no sea el 
principal objetivo de la sesión de EF. Es más, aquello puede ser justificado en los objetivos transversales del currículo. En el caso del nuevo currículo chileno, se hablaría de objetivos actitudinales de aprendizaje (OAA), que desde el punto de vista teórico son muy importantes para la formación ciudadana, pero en lo práctico son objetivos de segunda categoría, ya que no son parte de una evaluación sistematizada, ni de un tiempo concreto para su desarrollo y tampoco son vinculantes a la promoción del alumnado.

En concreto, en el actual currículo de EFyS de primero a sexto año básico, se identifican un OAA que aborda la igualdad de género y es el siguiente: "Promover la participación equitativa de hombres y mujeres en toda actividad física o deporte" (Ministerio de Educación, 2013, p. 166). Sin embargo, en el currículo de EFyS para el alumnado de séptimo año básico a segundo año medio, ningún OAA hace alusión al tema (Ministerio de Educación, 2016), de modo que se aprecia una descontinuación de la temática en una etapa muy importante, como es la adolescencia.

En cuanto a la inclusión educativa, se identifica un OAA vinculado en el currículo de EFyS de primer a sexto año básico, que señala lo siguiente: "Respetar la diversidad física de las personas, sin discriminar por características como altura, peso, color de piel o pelo, etc." (Ministerio de Educación, 2013, p. 166). Este objetivo, deja en evidencia el enfoque mecanicista que se encuentra presente en el currículo, debido a que solamente se centra en aspectos biológicos y antropométricos, ignorando la diversidad de pensamiento o de sexualidad, que implica al sujeto en su totalidad. Cabe destacar, que ningún otro OAA en esta etapa escolar hace referencia a los últimos aspectos mencionados. Luego el currículo de EFyS para séptimo año básico hasta segundo año medio, amplía aquel OAA, debido a que ya no solamente se centra en aspectos físicos, señalando lo siguiente: "Promover la participación de todos en las actividades físicas, valorando la diversidad de las personas, sin discriminar por características como altura, peso, color de piel, origen, condición física, discapacidades, etc.” (Ministerio de Educación, 2016, p. 259). No obstante, a pesar de que se aprecia una amplitud conceptual, se mantiene el sesgo de la perspectiva mecanicista, ya que existe una mayoría de ejemplos que aluden al aspecto físico y no se menciona la diversidad sexual, considerando que en Chile la comunidad LGTBI sufre constantemente 
de violencia homofóbica o transfóbica. Es tal la gravedad, que ante la brutal muerte del joven chileno Daniel Zamudio, que fue víctima de un ataque homofóbico, se aprobó una ley contra la discriminación que se conoce popularmente como la Ley Zamudio (Ley 20.609). Por lo tanto, es una gran irresponsabilidad que el currículo de EFyS no incluya explícitamente el respeto ante la diversidad sexual, porque se convierte en un silencio cómplice, pero que se comprende desde la colonización del ámbito educativo.

Como se ha mencionado, el profesorado cumple una labor fundamental en la interpretación del currículo escolar, de modo que son una gran contribución los estudios que abordan las creencias o teorías subjetivas del profesorado frente a la temática. Así, un estudio que abordó la opinión del profesorado chileno de una región del sur del país, sobre la clases mixtas de EF, señala que más profesoras que profesores se encuentra de acuerdo en que "las clases mixtas no desarrollan la igualdad para ambos sexos porque se acentúan las conductas machistas del alumnado masculino, las profesoras están más de acuerdo que los varones” (Castillo, Martínez-López y Zagalaz, 2010, p. 85). En cuanto a la justificación de aquellas docentes, se manifiesta que "las profesoras no están a favor de la realización de la clases en grupos mixtos debido a las conductas machistas existentes, porque las clases mixtas contribuirían a que las alumnas tiendan a tener actitudes masculinas" (Castillo, Martínez y Zagalaz, 2010, p. 85). Aquella opinión permite descubrir la mirada androcéntrica que se encuentra presente en el discurso de aquellas profesoras, ya que no hay ninguna actitud que esencialmente pueda ser atribuida al género femenino o masculino, como el patriarcado lo ha realizado por medio de los estereotipos de género. Es más, este es uno de los argumentos que se pueden utilizar para justificar la discriminación de las mujeres u hombres que no deseen reproducir la masculinidad o la feminidad hegemónica, tanto a nivel corporal, como a nivel motriz.

Este asunto de las creencias del profesorado de EFCh, fue investigado en el estudio de Moreno-Doña, Trigueros-Cervantes y Rivera-García (2014), que identificaron una relación dicotómica entre sus teorías implícitas, ya que muchas de ellas se enmarcan en un paradigma tradicional (repetición de estereotipos sociales) y otras en un paradigma sociocrítico, destacando como barreras para la transformación social, el cambio cultural, la 
situación curricular, la formación profesional y la desvalorización de la EF escolar. Asimismo, se indica que el trabajo orientado a trascender las desigualdades sociales muestra "cierta aspiración a una EF crítica, aunque muy superficial y fundamentada" (p. 94), lo cual expresa la necesidad de que el profesorado sea sometido a una profunda formación teórica en el tema, ya que de lo contrario, el deseo de cambiar aquellos habitus que perjudican la desigualdad de género y la inclusión educativa tendrá menos posibilidades de concretarse.

Por otra parte, un estudio español orientado a reducir la discriminación por sexo en la clase de EF, por medio de un programa compuesto por las técnicas del Speed dating, visionado de videos, mural de la igualdad de género y leyendas deportivas, identificó los siguiente: a) los chicos presentan mayor cantidad de conductas negativas por razón de sexo; b) los contenidos alternativos presentan mayor participación del alumnado; c) la agrupación por habilidad en los grupos mixtos favorece la igualdad de oportunidades entre los sexos; d) y la formación moral disminuye las actitudes y conductas discriminatorias (Ramos y Hernández, 2014). Un segundo estudio español en el que participó el alumnado que cursa la Licenciatura de Ciencias del Deporte y que recoge su opinión sobre la homofobia y el heterosexismo en la clase de EF escolar, describe entre sus hallazgos un sesgo negativo para los hombres, al igual que el estudio anterior, ya que son ellos los que utilizarían mayormente un lenguaje heterosexista y homofóbico. Además, declararían percibir una pasividad del profesorado ante esta problemática en la educación secundaria (Piedra et al., 2013).

\section{Consideraciones finales}

Considerando que reformular la EF, es algo indeseado para muchas personas, tanto especialistas como no especialistas en el área, porque se encuentran conformes con el orden social establecido y, por lo tanto, son indiferentes ante las injusticias sociales que se viven tanto en las naciones occidentales como orientales, cabe preguntarse, ¿es posible en la actualidad desarrollar una EFC en Chile? Al respecto, Vicente (2013), plantea que una EF enfocada desde esa perspectiva, requiere lo siguiente. 
Emprender una transformación de la educación física desde la perspectiva de la pedagogía crítica exige trascender, en todo caso, de la consideración de la educación física como un particular momento escolar en el que la experiencia es un mero proceso de construcción de la motricidad a la consideración de la educación física como un espacio político en el que la experiencia es un nudo de tensiones culturales; un nudo de tensiones sobre el que inciden y confluyen distintas operaciones disciplinarias (p. 325).

Tomando en cuenta aquel planteamiento, es posible señalar que mientras el currículo chileno y por consiguiente, de EF, mantenga una lógica tecnocrática o economicista de la educación, es muy complejo emprender procesos pedagógicos que mantengan una lógica crítica y emancipadora. Sin embargo, es posible desarrollar propuestas que estimulen la crítica sociocultural del alumnado e incentiven la transformación social, al mismo tiempo que se plantea una EF alternativa al modelo tradicional. Por esa razón, no se debe perder la esperanza ante las dificultades, sino que por el contrario, hay que continuar la lucha pedagógica y aportar con diferentes acciones que sean parte de un compromiso éticopolítico hacia la sociedad. Algunas de ellas, son las que plantea Brasó y Torrebadella (2018), como desarrollar prácticas no sexistas, fomentar el cuidado del medio ambiente, prácticas a favor de la paz, el respeto por los derechos humanos y ante todo, estimular la reflexión del alumnado. En cuanto a algunas acciones concretas para que el profesorado de EF pueda contribuir a la disminución de la discriminación, se recomienda incluir contenidos alternativos que no se caractericen por los estereotipos de género y si se trabaja con aquellos contenidos socialmente estereotipados, no permitir que el alumnado determine su agrupación, para de esta forma garantizar grupos mixtos con niveles homogéneos de competencia motriz (Ramos y Hernández, 2014).

Finalmente, se concluye que los antecedentes demuestran la importancia de que en Chile se realicen más estudios sobre la materia, pero lo más importante, es que se genere una profunda sensibilización del tema con el profesorado en formación, como con el profesorado en ejercicio (formación permanente), para de esta forma contribuir a la comprensión de estos fenómenos sociales. En términos más ambiciosos, sería ideal que el currículo nacional de EFCh asuma un carácter más socio-crítico, para que aporte 
responsablemente a transformar las desigualdades y discriminaciones sufridas por el alumnado.

\section{Referencias bibliográficas}

Andrónicos, M., Yaluris, N., Kakridís, I., Karayorga-Stazakopulu, Z., Kirkos, V., Paleologos, K.,... Sakelarakis, I. (2003). Los Juegos Olímpicos en la Grecia Antigua. Atenas: Ekdotiké Athenon.

Apple, M. (2011). Democratic education in neoliberal and neoconservative times. International Studies in Sociology of Education, 21(1), 21-31.

Bourdieu, P. (2000). La dominación masculina. Barcelona: Anagrama.

Carter-Thuillier, B. y Moreno, A. (2017). Globalización económica, postmodernidad y sistema educativo: contradicciones y alternativas desde una Educación Física crítica. Estudios Pedagógicos, 43(3), 103-117.

Carrillo, I. (2017). Los nudos del género. Apuntes para la formación ética de educadoras y educadores. Revista Internacional de Educación para la Justicia Social, 6(2), 2948.

Castillo, M., Martínez-López, E. y Zagalaz, M. (2010). Análisis de la opinión del profesorado de Educación Física de la Región de Los Lagos (Chile), sobre el desarrollo de las clases en torno a una modalidad mixta de enseñanza. Journal of Sport and Health Research, 2(2), 77-94.

Cavieres, E. y Apple, M. (2016). Le ley docente y la clase media: controlando el desarrollo de los profesores chilenos. Cadernos Cedes, 36(100), 265-280.

Chomsky, N. (2003). La (Des)educación. Barcelona: Crítica.

Curieses, P. (2017). Por una escuela inclusiva. Las fronteras del género. Revista Internacional de Educación para la Justicia Social, 6(2), 63-79.

Devís, J. (2008). El juego en el currículum de la Educación Física: aproximación crítica (o el reino de lo posible en la postmodernidad). Educación Física y Deporte, 27(2), 7989. 
Díez, E. (2010). La globalización neoliberal y sus repercusiones en educación. Revista electrónica interuniversitaria de formación del profesorado, 13(2), 23-38.

Fernández-Balboa, J. (1993). Aspectos crítico y cívico del rol de los/las profesionales de la Educación Física y el Deporte: Conexiones con la política, la economía y el medio ambiente. Apunts. Educación Física y Deportes, 34, 74-82.

Freire, P. (1975). Pedagogía del Oprimido. Madrid: Siglo veintiuno.

Freire, P. (2001). Política y Educación. Buenos Aires: Siglo veintiuno.

Giroux, H. (1999). Pedagogía crítica como proyecto de profecía ejemplar. Cultura y política en el nuevo milenio. En F. Imbernón (Ed.), La educación en el siglo XXI. Los retos del futuro inmediato (pp. 53-62). Barcelona: Graó.

Jordi, J. y Torrebadella, X. (2018). Reflexiones para (re)formular una educación física crítica. Revista Internacional de Medicina y Ciencias de la Actividad Física y el Deporte, 18(71), 441-462.

Ministerio de Educación. (2013). Bases curriculares. Educción Básica. Santiago: República de Chile.

Ministerio de Educación. (2016). Bases curriculares. $7^{o}$ básico a $2^{o}$ medio. Santiago: República de Chile.

Moreno, A., Marlen, C., Almonacid, A., y Vargas, A. (2013). La educación física chilena. Un modelo tecnocrático de la enseñanza y desvalorización del colectivo docente. Tandem. Didáctica de la Educación Física, 13(42), 7-17.

Moreno-Doña, A., Trigueros-Cervantes, C. y Rivera-García, E. (2013). Percepciones sobre la autoevaluación en la formación de profesores de educación física. Revista Internacional de Medicina y Ciencias de la Actividad Física y el Deporte, 13(52), 719-735.

Moreno, A., Gamboa, R. y Poblete, C. (2014). La educación física en Chile: Análisis crítico de la documentación ministerial. Revista Brasileira de Ciências do Esporte, 36(2), 411-427. 
Moreno, A. y Gamboa, R. (2014). Dictadura chilena y sistema escolar: "a otros dieron de verdad esa cosa llamada educación”. Educar em Revista, 51, 51-66.

Moreno-Doña, A., Trigueros-Cervantes, C. y Rivera-García, E. (2014). La Educación Física en Chile: Un análisis de las creencias del profesorado de la enseñanza primaria y secundaria. Movimento, 20(Esp.), 81-96.

Moreno, A. y Poblete, A. (2015). La educación física chilena y su profesorado: proponiendo algunos retos para la investigación en el área. Retos. Nuevas Tendencias en Educación Física, Deportes y Recreación, 28, 291-296.

Moreno, A. (2018). La Educación Física chilena en educación básica: Una caracterización crítica. Journal of the Latin American socio-cultural studies of sport, 9(2), 65-78.

Moreno, A., Toro, S. y Gómez-Gonzalvo, F. (2018). Crítica de la Educación Física Crítica: Eurocentrismo pedagógico y limitaciones epistemológicas. Psychology, Society, \& Education, 10(3), 349-362.

Mujica, F., y Concha, R. (2015). Objetivos de aprendizaje de primer a sexto año básico en Educación Física y Salud: Análisis crítico de la documentación ministerial. Revista Motricidad Humana, 16(1), 18-25.

Mujica, F., Orellana, N. y Concha, R. (2017). Emociones en la clase de Educación Física: revisión narrativa (2010-2016). Revista Ágora para la Educación Física y el Deporte, 19(1), 119-134.

Mujica, F. (2018). Las emociones en la educación física escolar. El aporte de la evaluación cualitativa. EmásF. Revista Digital de Educación Física, 51, 64-78.

Mujica, F. y Orellana, N. (2018). Autopercepción de la vocación en docentes de educación física escolar en Chile. CPU-e. Revista de Investigación Educativa, 27, 203-229.

Oliva, M. (2008). Política educativa y profundización de la desigualdad en Chile. Estudios Pedagógicos, 34(2), 207-226.

Oliva, M. y Gascón, F. (2016). Estandarización y racionalidad política neoliberal: bases curriculares de Chile. Cadernos Cedes, 36(100), 301-318. 
Piedra, J., Rodríguez, A., Ries, F. y Ramírez, G. (2013). Homofobia, heterosexismo y educación física: Percepciones del alumnado. Revista de Currículum y Formación del Profesorado, 17(1), 325-338.

Puiggrós, A. (2010). Educación y sociedad en América Latina de fin de siglo: del liberalismo al neoliberalismo pedagógico. Estudios Interdisciplinarios de América Latina y el Caribe, 10(1), 1-1.

Ramírez, R. (2008). La pedagogía crítica. Una manera ética de generar procesos educativos. Folios, 28, 108-119.

Ramos, F. y Hernández, A. (2014). Intervención para la reducción de la discriminación por sexo en las clases de Educación Física según los contenidos y agrupamientos utilizados. Revista Española de Educación Física y Deportes, 404, 27-38.

Rivas, A. (2015). América Latina después de PISA: lecciones aprendidas de la educación en siete países 2000-2015. Buenos Aires: CIPPEC.

Sergio, M. (1999). Motricidade Humana, um corte epistemológico. Lisboa: Instituto Piaget.

Toro, S. (2006). Conocimiento y motricidad humana, aproximaciones y desafíos. Pensamiento Educativo, 38, 62-74.

Toro, S. (2007). Una aproximación epistemológica a la didáctica de la motricidad desde el discurso y práctica docente. Estudios Pedagógicos, 33(1), 29-43. doi: $10.4067 / \mathrm{S} 0718-07052007000100002$

Toro, S. y Valenzuela, P. (2012). Desde la acción a la enacción. Más allá del movimiento y la Educación Física. Estudios Pedagógicos, 38(Esp.), 211-230. doi: 10.4067/S071807052012000400012

Vidiella, J., Herraiz, F., Hernándz, F. y Sancho, J. (2010). Masculinidad hegemónica, deporte y actividad física. Movimento, 16(4), 93-115.

Vicente, M. (2013). Crítica de la educación física y Educación Física Crítica. Estado (crítico) de la cuestión. Movimento, 19(1), 309-329. 